University of Rhode Island

DigitalCommons@URI

Open Access Dissertations

1998

\title{
Boundedness, Periodicity and Stability of Some Difference Equations
}

Amal M. Al-Amleh

University of Rhode Island

Follow this and additional works at: https://digitalcommons.uri.edu/oa_diss

\section{Recommended Citation}

Al-Amleh, Amal M., "Boundedness, Periodicity and Stability of Some Difference Equations" (1998). Open Access Dissertations. Paper 544.

https://digitalcommons.uri.edu/oa_diss/544

This Dissertation is brought to you for free and open access by DigitalCommons@URI. It has been accepted for inclusion in Open Access Dissertations by an authorized administrator of DigitalCommons@URI. For more information, please contact digitalcommons-group@uri.edu. 


\section{BOUNDEDNESS, PERIODICITY, AND STABILITY \\ OF SOME DIFFERENCE EQUATIONS}

\section{BY}

AMAL M. AL-AMLEH

4

A DISSERTATION SUBMITTED IN PARTIAL FULFILLMENT OF THE REQUIREMENTS FOR THE DEGREE OF DOCTOR OF PHILOSOPHY

IN

APPLIED MATHEMATICAL SCIENCES

UNIVERSITY OF RHODE ISLAND 
DOCTOR OF PHILOSOPHY DISSERTATION

OF

AMAL M. AL-AMLEH

APPROVED:

Dissertation committee

-

Major Professor Gerasimes Lades

Edward a Mreve

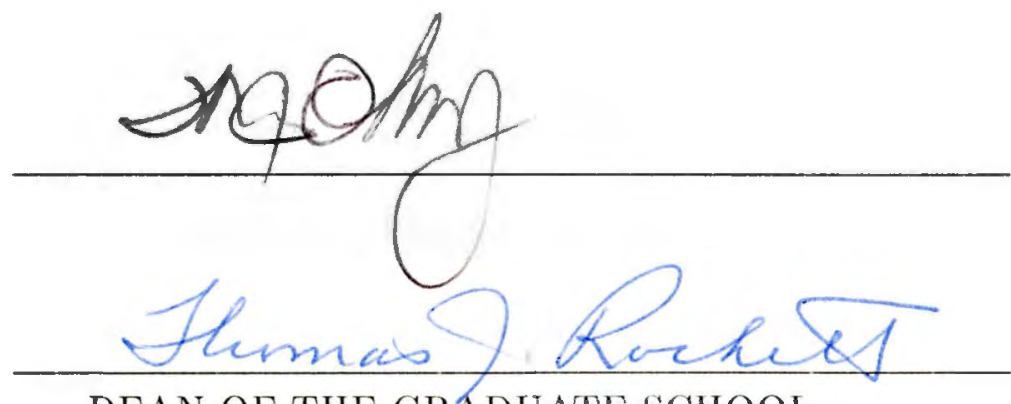

DEAN OF THE GRADUATE SCHOOL

UNIVERSITY OF RHODE ISLAND 


\section{ABSTRACT}

We investigate the boundedness character, the periodic nature, and the stability behavior of solutions of three difference equations.

The first equation is

$$
x_{n+1}=\max \left\{\frac{A}{x_{n}}, \frac{B}{x_{n-1}}\right\}, \quad n=0,1, \ldots
$$

where the parameters $A$ and $B$ and the initial conditions $x_{-1}$ and $x_{0}$ are nonzero real numbers. We obtain necessary and sufficient conditions for every solution to be eventually periodic. We also give a precise description of the period in terms of $A$, $B$, and the initial conditions.

The second equation is

$$
x_{n+1}=\frac{x_{n}+x_{n-1} x_{n-2}}{x_{n} x_{n-1}+x_{n-2}}, \quad n=0,1, \ldots
$$

where the intial conditions $x_{-2}, x_{-1}$, and $x_{0}$ are positive real numbers. We present a detailed analysis of the semicycles of solutions and show that the equilibrium of the equation is globally asymptotically stable. We also extend the global stability results to a general class of difference equations of the form

$$
x_{n+1}=f\left(x_{n}, x_{n-1}, \ldots, x_{n-k}\right), n=0,1, \ldots
$$

where the function $f$ satisfies the strong negative feedback property. 
Finally we investigate the boundedness character of the positive solutions of the Plant-Herbivore System

$$
\left.\begin{array}{l}
x_{n+1}=\frac{\alpha x_{n}}{\beta x_{n}+e^{y_{n}}} \\
y_{n+1}=\gamma\left(x_{n}+1\right) y_{n}
\end{array}\right\}, n=0,1, \ldots
$$

where $\alpha \in(1, \infty), \beta \in(0, \infty)$, and $\gamma \in(0,1)$ and the intial conditions $x_{0}$ and $y_{0}$ are arbitrary positive numbers. 


\section{ACKNOWLEDGEMENT}

I would like to express my deep gratitude to my advisor Professor Gerry Ladas, for his guidance, help, and encouragement. Without his mentoring, this work would not be possible. I would also like to thank Professor Ed. Grove and my co-authors Dr. Jeff Hoag and Nicole Kruse. 


\section{PREFACE}

This dissertation was prepared in accordance with the "University of Rhode Island Manuscript Plan" option. It is divided into two parts. Part I contains an introduction to our work and Part II, which is the main body of the dissertation, consists of three manuscripts that have been written in a form suitable for publication in international mathematical journals.

Manuscript I (co-authored with G. Ladas and J. Hoag) has been accepted for publication in Computers and Mathematics with Applications, Advances in Difference Equations II, 1998 (to appear).

Manuscript II (co-authored with G. Ladas and N. Kruse) has been accepted for publication in the Journal of Difference Equations and Applications (to appear).

Manuscript III (co-authored with G. Ladas and J. Hoag) has been submitted for publication.

The bibliography at the end of the dissertation refers to all sources mentioned throughout this dissertation. 


\section{Table of Contents}

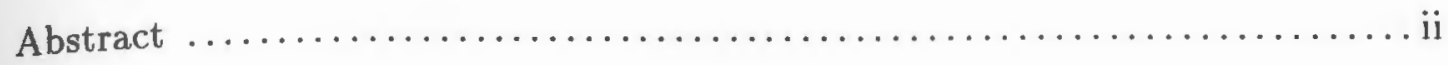

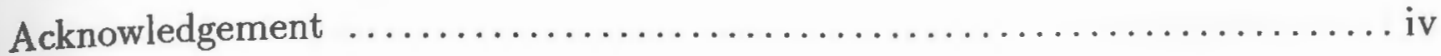

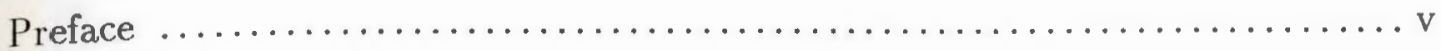

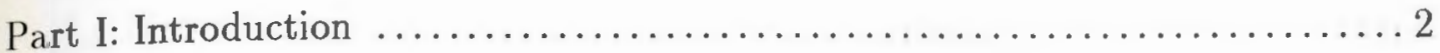

Part II: Manuscripts:

I: A Difference Equation with Eventually Periodic Solutions ............

II: On a Class of Difference Equations with Strong Negative

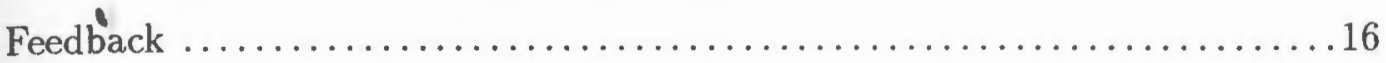

III: Boundedness of Solutions of a Plant-Herbivore System . . . . . . . . . 41

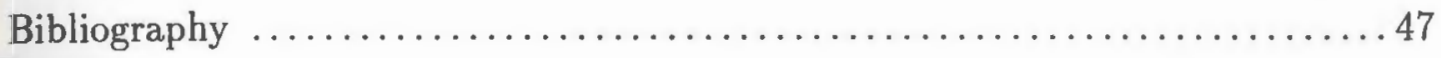


PART I 


\section{INTRODUCTION}

We investigate the boundedness character, the periodic nature, and the stability behavior of solutions of three difference equations.

The first equation is

$$
x_{n+1}=\max \left\{\frac{A}{x_{n}}, \frac{B}{x_{n-1}}\right\}, n=0,1, \ldots
$$

where the parameters $A$ and $B$ and the initial conditions $x_{-1}$ and $x_{0}$ are nonzero real numbers. We obtain necessary and sufficient conditions for every solution to be eventually periodic. We also give a precise description of the period in terms of $A, B$, and the initial conditions. This equation was discovered while looking for difference equations with the property that every solution is eventually periodic.

The second equation is

$$
x_{n+1}=\frac{x_{n}+x_{n-1} x_{n-2}}{x_{n} x_{n-1}+x_{n-2}} \quad, n=0,1, \ldots
$$

where the initial conditions $x_{-2}, x_{-1}$, and $x_{0}$ are positive real numbers. We persent a detailed analysis of the semicycles of solutions and show that the equilibrium of the equation is globally asymptotically stable. Motivated by our experience with this example, we extend the global stability results to a general class of difference equations of the form

$$
x_{n+1}=f\left(x_{n}, x_{n-1}, \ldots, x_{n-k}\right), \quad n=0,1, \ldots
$$

where the function $f$ satisfies the strong negative feedback property defined as 
follows:

Definition We say that a function $f\left(x_{0}, x_{1}, \ldots, x_{k}\right)$ with a unique positive fixed point $\bar{x}$ satisfies the strong negative feedback property with respect to the variable $x_{i_{0}}$, for some $i_{0} \in\{0,1, \ldots, k\}$, if

$$
f \in C\left[(0, \infty)^{k+1},(0, \infty)\right]
$$

and

$$
\text { - }\left[x_{i_{0}}-f\left(x_{0}, x_{1}, \ldots, x_{k}\right)\right]\left[\frac{\bar{x}^{2}}{x_{i_{0}}}-f\left(x_{0}, x_{1}, \ldots, x_{k}\right)\right] \leq 0
$$

for all $x_{0}, x_{1}, \ldots, x_{k} \in(0, \infty)$, with equality if and only if $x_{i_{0}}=\bar{x}$.

Finally we investigate the boundedness character of solutions of the following Plant-Herbivore System

$$
\left.\begin{array}{l}
x_{n+1}=\frac{\alpha x_{n}}{\beta x_{n}+e^{y_{n}}} \\
y_{n+1}=\gamma\left(x_{n}+1\right) y_{n}
\end{array}\right\} \quad, n=0,1, \ldots
$$

where $\alpha \in(1, \infty), \beta \in(0, \infty)$, and $\gamma \in(0,1)$ and the initial condtions $x_{0}$ and $y_{0}$ are arbitrary positive numbers. This system was introduced and studied by Allen, Hannigan, and Strauss. The model describes the interaction of the grape vine and the adult apple twig borer. 
PART II 
MANUSCRIPT I 


\title{
A DIFFERENCE EQUATION WITH EVENTUALLY PERIODIC SOLUTIONS
}

\begin{abstract}
We show that every solution of the difference equation

$$
x_{n+1}=\max \left\{\frac{1}{x_{n}}, \frac{A}{x_{n-1}}\right\}, \quad n=0,1, \ldots
$$

where the parameter $\mathrm{A}$ and the initial conditions $x_{-1}$ and $x_{0}$ are nonzero real numbers is eventually periodic with period 2,3 , or 4 . We also give a precise description of the period in terms of $\mathrm{A}$ and the initial conditions.
\end{abstract}




\section{Introduction}

Consider the difference equation

$$
x_{n+1}=\max \left\{\frac{1}{x_{n}}, \frac{A}{x_{n-1}}\right\}, \quad n=0,1, \ldots
$$

where the parameter $A$ and the initial conditions $x_{-1}$ and $x_{0}$ are nonzero real numbers. We show that every solution of Eq.(1) is eventually periodic with period 2, 3, or 4 and we determine the period in terms of $A$ and the initial conditions.

Another example of a difference equation with the property that every solution is eventually periodic is

$$
x_{n+1}=\left\{\begin{array}{ll}
\frac{x_{n}+x_{n-1}}{2} & \text { if }\left(x_{n}+x_{n-1}\right) \text { is even } \\
x_{n}-x_{n-1} & \text { if }\left(x_{n}+x_{n-1}\right) \text { is odd }
\end{array}, n=0,1, \ldots\right.
$$

with initial conditions in the set $\mathbf{Z}$ of all integers. Eq.(2) was investigated by Clark and Lewis [1] who proved that every solution is eventually constant or eventually an integral multiple of the six cycle $\{1,3,2,-1,-3,-2\}$.

It was also conjectured in [2] that every solution of the difference equation

$$
x_{n+1}=\left\{\begin{array}{ll}
\frac{\alpha x_{n}+\beta x_{n-1}}{2} & \text { if }\left(\alpha x_{n}+\beta x_{n-1}\right) \text { is even } \\
\gamma x_{n}+\delta x_{n-1} & \text { if }\left(\alpha x_{n}+\beta x_{n-1}\right) \text { is odd }
\end{array}, n=0,1, \ldots\right.
$$


where $\alpha, \beta, \gamma, \delta \in\{-1,1\}$ and $x_{-1}, x_{0} \in \mathbf{Z}$, is eventually periodic in all cases except when

$$
\begin{aligned}
& \text { either } \quad \alpha=\beta=\gamma=\delta=1 \\
& \text { or } \quad-\alpha=\beta=-\gamma=\delta=1 .
\end{aligned}
$$

Furthermore the only possible periods of solutions of Eq.(3) are 1, 2, 3, 4, 5, 6, 8, or 10.

Finally we should mention the so-called $(3 x+1)$ conjecture according to which every solution of the difference equation

$$
x_{n+1}=\left\{\begin{array}{cl}
\frac{x_{n}}{2} & \text { if } x_{n} \text { is even } \\
\frac{3 x_{n}+1}{2} & \text { if } x_{n} \text { is odd }
\end{array} \quad, n=0,1, \ldots\right.
$$

with the initial condition $x_{0}$ being a positive integer, is eventually equal to the two cycle $\{1,2\}$. If $x_{0}$ is allowed to be an integer, then every solution of Eq.(4) is eventually equal to a cycle of length $1,2,3$, or 11 . For more information see [3].

\section{Main Results}

The main results about Eq.(1) are included in the following theorem.

Theorem 1 Assume that the parameter $A$ and the initial conditions $x_{-1}$ and $x_{0}$ are nonzero real numbers. Then every solution of Eq.(1) is eventually periodic with period 2, 3, or 4. More precisely the following are true. 
(i) Assume that $A<0$. Then every solution of Eq.(1) is eventually periodic with period two and is of the form $\{p, 1 / p\}$, for some positive number $p$ which depends on $A$ and the initial conditions $x_{-1}$ and $x_{0}$.

(ii) Assume that $A>0$ and the initial conditions $x_{-1}$ and $x_{0}$ are not both negative numbers. Then every solution of Eq.(1) is eventually periodic with period
(a) 2 if $A \in(0,1)$;
(b) 3 if $A=1$;
(c) 4 if $A \in(1, \infty)$.

(iii) Assume that $A>0$ and the initial conditions $x_{-1}$ and $x_{0}$ are both negative numbers. Then every solution of Eq.(1) is negative and eventually periodic with period
(a) 2 if $A \in(1, \infty)$;
(b) 3 if $A=1$;
(c) 4 if $A \in(0,1)$.

Proof. (i) It is easy to see that in this case, every solution of Eq.(1) is eventually positive. Hence Eq.(1) eventually becomes

$$
x_{n+1}=\frac{1}{x_{n}}
$$

from which the result follows. 
(ii) In this case also every solution of Eq.(1) is eventually positive and so without loss of generality we will assume that $x_{-1}, x_{0} \in(0, \infty)$.

(a) Set $x_{n}=A^{y_{n}}$. Then Eq.(1) becomes

$$
y_{n+1}=\min \left\{-y_{n}, 1-y_{n-1}\right\}, \quad n=0,1, \ldots
$$

where $y_{-1}, y_{0}$ are real numbers.

Let $\mathbf{S}=\left\{(\alpha, \dot{\beta}): \beta=-\alpha,-\frac{1}{2} \leq \alpha \leq \frac{1}{2}\right\}$ and $\mathbf{L}=\{(\alpha, \beta): \beta=-\alpha, \alpha \in \mathbf{R}\}$. Set $y_{-1}=\alpha$ and $y_{0}=\beta$.

Case I: $(\alpha, \beta) \in \mathbf{S}$. Then one can see that every solution of Eq.(5) is periodic with period two of the form $\{\alpha,-\alpha\}$.

Case II: $(\alpha, \beta) \notin \mathbf{S}$. Now we claim that after a finite number of iterations, the orbit of the solution will intersect with $\mathbf{L}$. Clearly every solution of Eq.(5) oscillates and so without loss of generality we may assume that $\alpha \geq 0$ and $\beta \leq 0$. Then the solution takes one of the following forms:

$$
\begin{gathered}
\alpha, \beta,-\beta, \ldots ; \\
\alpha, \beta, 1-\alpha, \alpha-1, \ldots ; \\
\alpha, \beta, 1-\alpha, 1-\beta, \beta-1, \ldots .
\end{gathered}
$$


Note that in each case the orbit of the solution has intersected with $\mathbf{L}$. It remains to show that if the solution starts on $(\mathbf{L}-\mathbf{S})$, then eventually its orbit will be on $\mathbf{S}$. To this end, let $(\alpha, \beta) \in(\mathbf{L}-\mathbf{S})$. Then the solution is $\{\alpha,-\alpha, 1-\alpha, \alpha-1,1-\alpha, \ldots\}$. Note that $(\alpha,-\alpha) \in \mathbf{L}$ and $(\alpha-1,1-\alpha) \in \mathbf{L}$ and the distance between these two points is $\sqrt{2}$. Furthermore the point $(\alpha-1,1-\alpha)$ is closer to $\mathbf{S}$ than the point $(\alpha,-\alpha)$. So after a finite number of iterations, the orbit of the solution will be on $\mathbf{S}$.

(b) It is easy to see that every solution of Eq.(1) contains two consecutive terms both $\geq 1$. So it suffices to consider the case when $x_{-1} \geq 1$ and $x_{0} \geq 1$. Then clearly the solution becomes either $\left\{x_{-1}, x_{0}, \frac{1}{x_{-1}}, x_{-1}, x_{-1}, \frac{1}{x_{-1}}, x_{-1}, \ldots\right\}$ or $\left\{x_{-1}, x_{0}, \frac{1}{x_{0}}, x_{0}, x_{0}, \frac{1}{x_{0}}, \ldots\right\}$, and $s o$ in either case the solution is periodic with period three.

(c) Let $x_{n}=A^{y_{n}+\frac{1}{2}}$. Then Eq.(1) becomes

$$
y_{n+1}=\max \left\{-1-y_{n},-y_{n-1}\right\}, \quad n=0,1, \ldots
$$

where $y_{-1}$ and $y_{0}$ are real numbers. It is easy to see that every solution of Eq.(6) contains two consecutive non-negative terms.

Let $\mathbf{B}=\{(\alpha, \beta):|\alpha|+|\beta| \leq 1\}$ and $\mathbf{T}=\{(\alpha, \beta): \beta=-\alpha-1, \alpha \leq 0\}$. Set $y_{-1}=\alpha$ and $y_{0}=\beta$. 
Case I: $(\alpha, \beta) \in \mathbf{B}$. Then one can see that every non-trivial solution of Eq.(6) is periodic with period 4 of the form $\{\alpha, \beta,-\alpha,-\beta\}$.

Case II: $(\alpha, \beta) \notin \mathbf{B}$. We now claim that after a finite number of iterations, the orbit of the solution will intersect with $\mathbf{T}$. To see this, without loss of generality assume that $\alpha \geq 0$ and $\beta \geq 0$. Then the solution becomes either $\{\alpha, \beta,-\alpha, \alpha-1, \ldots\}$ or $\{\alpha, \beta,-1-\beta, \ldots\}$ which proves our claim.

Finally observe that if $(\alpha, \beta) \in(\mathbf{T}-\mathbf{B})$, then $\alpha<-1$ and the solution is

$$
\{\alpha,-1-\alpha,-\alpha, 1+\alpha,-2-\alpha, \ldots\}
$$

Note that $(1+\alpha,-2-\alpha) \in \mathbf{T}$ and the distance between $(\alpha,-1-\alpha)$ and $(1+\alpha,-2-\alpha)$ is $\sqrt{2}$. Furthermore the point $(1+\alpha,-2-\alpha)$ is closer to $\mathbf{B}$ than the point $(\alpha,-1-\alpha)$. Hence after a finite number of iterations, the orbit of the solution will be in $\mathbf{B}$.

(iii) In this case the change of variables $x_{n}=-y_{n}$ reduces Eq.(1) to

$$
y_{n+1}=\min \left\{\frac{1}{y_{n}}, \frac{A}{y_{n-1}}\right\}, \quad n=0,1, \ldots
$$

with positive initial conditions. The remaining part of the proof is similar to that given in part (ii) and will be omitted. 
Remark It is interesting to note that the more general coplation

$$
x_{n+1}=\max \left\{\frac{a}{x_{n}}, \frac{A}{x_{n-1}}\right\}, \quad n=0,1 \ldots
$$

where $a, A$ and the initial conditions $x_{-1}$ and $x_{0}$ are nonzero real numbers, does not have the property that every solution is eventually periodic. In fact if $a=-1$ and $A=-2$, then the solution with the initial conditions $x_{-1}=2$ and $x_{0}=-1 / 2$ is unbounded and is given by

$$
\text { - } \quad 2, \frac{-1}{2}, 2, \ldots, 2^{n}, \frac{-1}{2^{n}}, 2^{n}, \ldots
$$

In general, one can show that if $a$ and $A$ are both negative and not equal, then every solution of Eq.(7) with initial conditions in $\mathbf{R}-\{0\}$ is mulrounded.

\section{References}

[1] Clark, D. and Lewis, J.T., A Collatz-Type Difference Equation, Congressus Numerantium 111 (1995), 129-135.

[2] Ladas, G.. Open Problems and Conjectures,J. Diff. Equa. arı.d Appl. 2 (1) (1996), 97-99.

[3] Lagarias, J.G., The $(3 x+1)$ problem and its generalizalions, Amer. Ifath. Monthly 92 (1985), 3-23. 
MANUSCRIPT II 


\title{
ON A CLASS OF DIFFERENCE EQUATIONS WITH STRONG NEGATIVE FEEDBACK
}

\begin{abstract}
We investigate the behavior of the positive solutions of a class of difference equations with strong negative feedback. In particular we show that the positive equilibrium is globally asymptotically stable.
\end{abstract}




\section{Introduction}

Computer observations predicted that the positive equilibrium of each of the following three difference equations

$$
\begin{aligned}
& x_{n+1}=\frac{x_{n}+x_{n-1} x_{n-2}}{x_{n} x_{n-1}+x_{n-2}}, \quad n=0,1, \ldots, \\
& x_{n+1}=\frac{x_{n-1}+x_{n} x_{n-2}}{x_{n} x_{n-1}+x_{n-2}}, \quad n=0,1, \ldots,
\end{aligned}
$$

and

$$
\text { - } \quad x_{n+1}=\frac{x_{n}+x_{n-1} x_{n-2}}{x_{n} x_{n-2}+x_{n-1}}, \quad n=0,1, \ldots
$$

with positive initial conditions $x_{-2}, x_{-1}$ and $x_{0}$, is globally asymptotically stable. For the first two equations we present a detailed analysis of the semicycles of solutions and in all three cases we show that indeed the positive equilibrium $\bar{x}=1$ is globally asymptotically stable. For some motivation related to this problem see [1].

Unfortunately we found it very difficult to obtain a complete understanding of the semicycles of solutions of Eq.(3). This still remains an open problem. Fortunately, however, motivated by our experience dealing with Eqs.(1) and (2), we discovered that each of these three equations possesses a strong negative feedback property which can be used to establish the global attractivity of the equilibrium solution for each of the above three equations and for a general class of difference equations of the form

$$
x_{n+1}=f\left(x_{n}, x_{n-1}, \ldots, x_{n-k}\right), n=0,1, \ldots
$$

where the function $\mathrm{f}$ satisfies the strong negative feedback property defined as follows: 
Definition 1 We say that a function $f\left(x_{0}, x_{1}, \ldots, x_{k}\right)$ with a unique positive fixed point $\bar{x}$ satisfies the strong negative feedback property with respect to the variable $\mathbf{x}_{\mathbf{i}_{0}}$, for some $i_{0} \in\{0,1, \ldots, k\}$, if

$$
f \in C\left[(0, \infty)^{k+1},(0, \infty)\right]
$$

and

$$
\begin{gathered}
{\left[x_{i_{0}}-f\left(x_{0}, x_{1}, \ldots, x_{k}\right)\right]\left[\frac{\bar{x}^{2}}{x_{i_{0}}}-f\left(x_{0}, x_{1}, \ldots, x_{k}\right)\right] \leq 0} \\
\text { for all } x_{0}, x_{1}, \ldots, x_{k} \in(0, \infty)
\end{gathered}
$$

with equality if and only if $x_{i_{0}}=\bar{x}$.

For example for Eq.(3),

$$
f(x, y, z)=\frac{x+y z}{x z+y} \text { and } \bar{x}=1
$$

and one can show that $f$ satisfies the strong negative feedback property with respect to the variable $z$. That is,

$$
[z-f(x, y, z)]\left[\frac{1}{z}-f(x, y, z)\right] \leq 0
$$

with equality if and only if $z=1$.

For Eq.(1),

$$
f(x, y, z)=\frac{x+y z}{x y+z} \text { and } \bar{x}=1
$$

and $f$ satisfies the strong negative feedback property with respect to the variable $y$.

Finally for Eq.(2),

$$
f(x, y, z)=\frac{y+x z}{x y+z} \text { and } \bar{x}=1
$$

and $f$ satisfies the strong negative feedback property with respect to the variable $x$. 


\section{Equation (1)}

In this section we investigate the behavior of solutions of Eq.(1) with positive initial conditions $x_{-2}, x_{-1}$ and $x_{0}$. In particular we present a detailed analysis of the semicycles of solutions and use it to show that the positive equilibrium $\bar{x}=1$ is globally asymptotically stable.

\subsection{Eventually Trivial Solutions}

Here we establish necessary and sufficient conditions for a positive solution of Eq.(1) to be eventually equal to 1 . The main result is summarized in the following lemma.

Lemma 1 A positive solution of Eq.(1) is eventually equal to 1 if and only if

$$
\left(1-x_{-1}\right)\left(1-x_{0}\right)\left(x_{-2}-x_{0}\right)=0
$$

Proof Assume (5) holds. Then one can easily see that the following is true:

(i) if $x_{0}=1$, then $x_{n}=1 \quad$ for $n \geq 2$;

(ii) if $x_{-1}=1$, then $x_{n}=1 \quad$ for $n \geq 1$;

(iii) if $x_{-2}=x_{0}$, then $x_{n}=1 \quad$ for $n \geq 3$.

Conversely, assume that

$$
\left(1-x_{-1}\right)\left(1-x_{0}\right)\left(x_{-2}-x_{0}\right) \neq 0
$$

We will establish the stronger statement that

$$
x_{n} \neq 1 \text { for any } n \geq 1 \text {. }
$$


To this end assume for the sake of contradiction that for some $N \geq 1$,

$$
x_{N}=1 \text { and that } x_{n} \neq 1 \text { for }-1 \leq n \leq N-1 .
$$

Then

$$
1=x_{N}=\frac{x_{N-1}+x_{N-2} x_{N-3}}{x_{N-1} x_{N-2}+x_{N-3}}
$$

which implies that

$$
x_{N-1}=x_{N-3}
$$

and because of (6), $N \geq 2$. Therefore,

$$
x_{N-3}=x_{N-1}=\frac{x_{N-2}+x_{N-3} x_{N-4}}{x_{N-2} x_{N-3}+x_{N-4}}
$$

or equivalently,

$$
\left(1-x_{N-3}^{2}\right) x_{N-2}=0
$$

which is impossible.

Remark 1 If the initial conditions do not satisfy the equality (5), then $x_{n} \neq 1$ and $x_{n-1} \neq x_{n+1}$ for $n \geq-1$.

\subsection{Properties of Solutions which are not Eventually Trivial}

The main result in this section is the following lemma.

Lemma 2 Let $\left\{x_{n}\right\}_{n=-2}^{\infty}$ be a positive solution of Eq.(1) which is not eventually equal to 1 . Then the following inequalities are satisfied:

(i) $\left(1-x_{n+1}\right)\left(1-x_{n-1}\right)\left(x_{n}-x_{n-2}\right)<0$, for $n \geq 0$; 
(ii) $\left(1-x_{n-1}\right)\left(x_{n+1}-x_{n-1}\right)>0$, for $n \geq 0$;

(iii) $\left(1-x_{n+1}\right)\left(1-x_{n-1}\right)\left(1-x_{n-2}\right)<0$, for $n \geq 1$.

Proof Inequalities (i) and (ii) are immediate consequences of the identities

$$
1-x_{n+1}=-\frac{\left(1-x_{n-1}\right)\left(x_{n}-x_{n-2}\right)}{x_{n} x_{n-1}+x_{n-2}}, \quad \text { for } n \geq 0
$$

and

$$
x_{n+1}-x_{n-1}=\frac{x_{n}\left(1-x_{n-1}^{2}\right)}{x_{n} x_{n-1}+x_{n-2}}, \quad \text { for } n \geq 0
$$

respectively. Also, inequality (iii) is a consequence of (i) and (ii).

\subsection{Semicycle Analysis of Nontrivial Solutions}

For the investigation of the nontrivial solutions we need the following definitions.

Definition $2 A$ positive semicycle of a solution $\left\{x_{n}\right\}_{n=-2}^{\infty}$ of Eq. (1) consists of a "string" of terms $\left\{x_{l}, x_{l+1}, \ldots, x_{m}\right\}$, all of them greater than 1 , with $l \geq-2$ and $m \leq \infty$ such that

$$
\text { either } l=-2 \text {, or } l>-2 \text { and } x_{l-1}<1
$$

and

$$
\text { either } m=\infty \text {, or } m<\infty \text { and } x_{m+1}<1 \text {. }
$$

A negative semicycle of a solution $\left\{x_{n}\right\}_{n=-2}^{\infty}$ of Eq. (1) consists of a "string" of terms $\left\{x_{l}, x_{l+1}, \ldots, x_{m}\right\}$, all of them less than 1 , with $l \geq-2$ and $m \leq \infty$ such that

$$
\text { either } l=-2 \text {, or } l>-2 \text { and } x_{l-1}>1
$$


and

$$
\text { either } m=\infty \text {, or } m<\infty \text { and } x_{m+1}>1 \text {. }
$$

Note that, if $x_{-2}, x_{-1}, x_{0} \in(1, \infty)$, then $x_{1}>1$ if and only if $x_{0}<x_{-2}$. In fact, if

$$
x_{-2}, x_{-1}, x_{0} \in(1, \infty) \text { and } x_{0}<x_{-2}
$$

then by Lemma 2 ,

$$
1<x_{n}<x_{n-2}, \quad \text { for } n \geq 0
$$

and clearly the subsequences of even and odd terms of the solution decrease monotonically to 1 . Also by Lemma 2 (ii) and (iii), if for some $N \geq-1$,

$$
x_{N}, x_{N+1}, x_{N+2} \in(1, \infty)
$$

then one can see that, in fact, (7) holds. Therefore except perhaps for the first emicycle, a positive semicycle of a solution contains at most two terms. Concerning negative semicycles, it follows again by Lemma 2 (iii), that except perhaps for the first semicycle, a negative semicycle contains at most three terms. More precisely, the following result holds.

Lemma 3 Consider a solution of Eq.(1) which is not eventually greater than or equal to 1. Then with the possible exception of the first semicycle, the following statements are true:

- Every positive semicycle consists of one or two terms.

- Every negative semicycle consists of one or three terms. 
- Every positive semicycle of length two is followed by a negative semicycle of length one.

- Every positive semicycle of length one is followed by a negative semicycle of length three.

- Every negative semicycle of length three is followed by a positive semicycle of length two.

- Every negative semicycle of length one is followed by a positive semicycle of length òne.

Proof We have just seen that a positive semicycle of a solution which is not eventually greater than or equal to 1 , has one or two terms and a negative semicycle has at most three terms. Clearly, one of the following cases must occur for some $N \geq 0$ :

Case 1: $x_{N-2}<1, x_{N-1}>1$ and $x_{N}>1$.

In view of inequality (iii) of Lemma $2, x_{N+1}<1, x_{N+2}>1$, and $x_{N+3}<1$. Therefore a positive semicycle of length 2 is followed by a negative semicycle of length 1 , which in turn is followed by a positive semicycle with exactly one term.

Case 2: $x_{N-2}<1, x_{N-1}>1$ and $x_{N}<1$.

Again in view of inequality (iii) of Lemma $2, x_{N+1}<1, x_{N+2}<1$ and $x_{N+3}>1$. Furthermore $x_{N+4}>1$. Therefore a positive semicycle of length one, is followed by a negative semicycle of length three which in turn is followed by a positive semicycle containing exactly two terms. 


\subsection{Global Asymptotic Stability of the Equilibrium of Eq.(1)}

Here we show that the positive equilibrium of Eq.(1) is globally asymptotically stable.

Theorem 1 The positive equilibrium of Eq.(1) is globally asymptotically stable.

Proof The linearized equation of Eq.(1) about the positive equilibrium is

$$
y_{n+1}=0 \cdot y_{n}+0 \cdot y_{n-1}+0 \cdot y_{n-2}
$$

and so clearly, 1 is locally asymptotically stable. It remains to show that every positive solution $\left\{x_{n}\right\}_{n=-2}^{\infty}$ of Eq.(1) converges to 1 as $n \rightarrow \infty$. In the previous section we saw that this is true if the solution is eventually greater than or equal to 1 . We also saw that it is impossible for a solution to be eventually less than 1 . Hence it remains to consider solutions which are not eventually trivial and which oscillate about 1 . To simplify the proof we use without loss of generality the following notation. We denote by $x_{N-2}$ the term of a negative semicycle of length one, followed by $x_{N-1}$ which is the term of a positive semicycle of length one. Afterwards there is the negative semicycle: $x_{N}, x_{N+1}, x_{N+2}$, in turn followed by the positive semicycle: $x_{N+3}, x_{N+4}$, and so on. We now claim that the following statements are true:

(i) $x_{N}<x_{N+2}, x_{N+4}>x_{N+6}$, and $x_{N+5}<x_{N+7}$;

(ii) $x_{N+1} x_{N+3}<1$;

(iii) $x_{N+3} x_{N+5}>1$; 
(iv) $x_{N+8}>x_{N+5}$;

(v) $x_{N+4} x_{N+7}>1$;

(vi) $x_{N+2} x_{N+4}<1$.

Indeed the inequalities (i), (ii), (iii), and (vi) are straightforward. To show (iv), observe that $x_{N+7}>x_{N+5}$ and so

$$
x_{N+8}=\frac{x_{N+7}+x_{N+6} x_{N+5}}{x_{N+7} x_{N+6}+x_{N+5}}>\frac{x_{N+5}\left(1+x_{N+6}\right)}{x_{N+6}+1}=x_{N+5} .
$$

Finally, $x_{N+6}<x_{N+4}$ and so

$$
x_{N+7}=\frac{x_{N+6}+x_{N+5} x_{N+4}}{x_{N+6} x_{N+5}+x_{N+4}}>\frac{1+x_{N+5}}{x_{N+4} x_{N+5}+x_{N+4}}=\frac{1}{x_{N+4}} .
$$

Combining the above inequalities we obtain

(vii) $x_{N}<x_{N+2}<\frac{1}{x_{N+4}}<x_{N+7}<x_{N+9}$, and

(viii) $x_{N+1}<\frac{1}{x_{N+3}}<x_{N+5}<x_{N+8}<x_{N+12}$.

Now, we investigate the behavior of the seven subsequences $\left\{x_{N+i+7 k}\right\}_{k=0}^{\infty}$ for $i=$ $0,1, \ldots, 6$. Set

$$
L_{0}=\lim _{k \rightarrow \infty} x_{N+7 k} \text { and } L_{1}=\lim _{k \rightarrow \infty} x_{N+1+7 k}
$$

which exist because of (vii) and (viii), respectively. Then 


$$
\begin{aligned}
& \lim _{k \rightarrow \infty} x_{N+2+7 k}=L_{0}, \quad \text { because of (vii), } \\
& \lim _{k \rightarrow \infty} x_{N+3+7 k}=\frac{1}{L_{1}}, \quad \text { because of (viii), } \\
& \lim _{k \rightarrow \infty} x_{N+4+7 k}=\frac{1}{L_{0}}, \quad \text { because of (vii), } \\
& \lim _{k \rightarrow \infty} x_{N+5+7 k}=L_{1}, \quad \text { because of (viii), and } \\
& \lim _{k \rightarrow \infty} x_{N+6+7 k}=\frac{1+L_{0} L_{1}^{2}}{L_{0}+L_{1}^{2}} .
\end{aligned}
$$

It suffices to show that $L_{0}=L_{1}=1$. To this end note that

$$
\quad x_{N+3+7 k}=\frac{x_{N+2+7 k}+x_{N+1+7 k} x_{N+7 k}}{x_{N+2+7 k} x_{N+1+7 k}+x_{N+7 k}}
$$

and so by taking limits on both sides,

$$
\frac{1}{L_{1}}=\frac{L_{0}+L_{1} L_{0}}{L_{0} L_{1}+L_{0}}=1
$$

Considering the subsequence $\left\{x_{N+4+7 k}\right\}_{k=0}^{\infty}$ we can show in a similar way that $L_{0}=1$.

$\square$

Remark 2 The proof of Theorem 1 was given by a detailed analysis of the semicycles of solutions of Eq.(1). In chapter 5 we present a general theorem which also establishes the global asymptotic stability of the positive equilibrium and for which we do not need to know the exact pattern of the semicycles.

\section{Equation (2)}

In this section we investigate the behavior of solutions of Eq.(2) with positive initial conditions $x_{-2}, x_{-1}$ and $x_{0}$. Here we also present a detailed analysis of the semicy- 
cles of solutions and use it to show that the positive equilibrium $\bar{x}=1$ is globally asymptotically stable.

\subsection{Eventually Trivial Solutions}

Here we establish necessary and sufficient conditions for a positive solution of Eq.(2) to be eventually equal to 1 . The main result is summarized in the following lemma.

Lemma $4 A$ positive solution of Eq.(2) is eventually equal to 1 if and only if

$$
\text { 1 } \quad\left(x_{-1}-x_{0}\right)\left(x_{-2}-x_{-1}\right)\left(x_{0}-1\right)=0
$$

The proof is similar to the proof of Lemma 1 for Eq.(1) and will be omitted.

Remark 3 If the initial conditions do not satisfy the equation (8), then $x_{n} \neq 1$ and $x_{n-1} \neq x_{n-2}$ for all $n \geq 0$.

\subsection{Properties of Solutions which are not Eventually Trivial}

The properties of not eventually trivial solutions are based on the following lemma.

Lemma 5 Let $\left\{x_{n}\right\}_{n=-2}^{\infty}$ be a positive solution of Eq.(2) which is not eventually equal to 1. Then the following inequalities are satisfied:

(i) $\left(1-x_{n+1}\right)\left(1-x_{n}\right)\left(x_{n-1}-x_{n-2}\right)<0$, for $n \geq 0$;

(ii) $\left(1-x_{n}\right)\left(x_{n+1}-x_{n}\right)>0$, for $n \geq 0$;

(iii) $\left(1-x_{n+1}\right)\left(1-x_{n}\right)\left(1-x_{n-2}\right)<0$, for $n \geq 1$.

The proof is similar to the proof of Lemma 2 for Eq.(1) and will be omitted. 


\subsection{Semicycle Analysis of Nontrivial Solutions}

With the help of Lemma 5 it can be shown that a solution of Eq.(2) is eventually greater than 1 if and only if it is always greater than 1 which is true if and only if

$$
x_{-2}>x_{-1}>x_{0}>1 \text {. }
$$

Furthermore in this case the solution is strictly monotonically decreasing to 1 .

The following lemma is also a consequence of the properties in Lemma 5.

Lemma 6 Consider a solution of Eq.(2) which is not eventually greater than 1 or equal to 1. 'Then with the possible exception of the first semicycle, the following statements are true:

- Every positive semicycle consists of one or two terms.

- Every negative semicycle consists of one or three trems.

- Every positive semicycle of length two is followed by a negative semicycle of length three.

- Every positive semicycle of length one is followed by a negative semicycle of length one.

- Every negative semicycle of length three is followed by a positive semicycle of length one.

- Every negative semicycle of length one is followed by a positive semicycle of length two.

The proof is along the lines of the proof of Lemıma 3 for Eq.(1) and will be omitted. 


\subsection{Global Asymptotic Stability of the Equilibrium of Eq.(2)}

Theorem 2 The positive equilibrium 1 of Eq.(2) is globally asymptotically stable.

Proof With the help of linearized stability it can easily be shown that the positive equilibrium of Eq.(2) is locally asymptotically stable.

It follows by Lemma 6 that all terms above the equilibrium, except perhaps the first three, are decreasing and all terms below the equilibrium, except perhaps the first three, are increasing. If we denote by ' $L$ ' the limit of the terms below the equilibrium and by ' $U$ ' the limit of the terms above the equilibrium, then

$$
U=\frac{L+L^{2}}{L^{2}+L}=1
$$

and

$$
L=\frac{L+U L}{U L+L}=1
$$

Hence $\bar{x}=1$ is a global attractor and the proof is complete.

Remark 4 As for Theorem 1 the proof of Theorem 2 was also given by a detailed analysis of the semicycles of solutions of Eq.(2). The general theorem which we will prove in chapter 5 and which establishes the global asymptotic stability of the positive equilibrium can also be applied in this case. 


\section{Equation (3)}

In this section we investigate the behavior of solutions of Eq.(3) with positive initial conditions $x_{-2}, x_{-1}$ and $x_{0}$. Here we give some details about the semicycles of solutions and then we show that the positive equilibrium is globally asymptotically stable.

Remark 5 In the sequel the definition for a positive semicycle includes the terms which are equal to the equilibrium.

\subsection{Eventually Trivial Solutions}

Lemma 7 A positive solution of Eq.(3) is eventually equal to 1 if and only if two of the initial conditions $x_{-2}, x_{-1}, x_{0}$ are equal to 1 .

The proof is similar to the proof of Lemma 1 of Eq.(1) and will be omitted.

\subsection{Properties of Solutions which are not Eventually Trivial}

Lemma 8 A positive solution of Eq.(3) consists of infinitely many terms equal to 1, more exactly every third term is equal to 1, after the first term is equal to1, if and only if

$$
\left(x_{-2}-1\right)\left(x_{-1}-1\right)\left(x_{0}-1\right)\left(x_{-1}-x_{0}\right)=0 .
$$

The proof is similar to the proof of Lemma 1 of Eq.(1) and will be omitted.

Lemma 9 Let $\left\{x_{n}\right\}_{n=-2}^{\infty}$ be a positive solution of Eq.(3) which is never equal to 1 . Then the following inequalities are satisfied: 
(i) $\left(1-x_{n+1}\right)\left(1-x_{n-2}\right)\left(x_{n}-x_{n-1}\right)<0$, for $n \geq 0$;

(ii) $\left(1-x_{n-2}\right)\left(x_{n+1}-x_{n-2}\right)>0$, for $n \geq 0$;

(iii) $\left(1-x_{n+1}\right)\left(x_{n}-x_{n-1}\right)\left(x_{n+1}-x_{n-2}\right)<0$, for $n \geq 1$;

(iv) $\left(x_{n-2} \cdot x_{n+1}-1\right)\left(x_{n-2}-1\right)>0$, for $n \geq 1$.

The proof is along the lines of the proof of Lemma 2 of Eq.(1) and will be omitted.

\subsection{Semicycle Analysis of Nontrivial Solutions}

With the help of Lemma 9 (i) the following lemma about the properties of the semicycles of nontrivial solutions can be established.

Lemma 10 Consider a solution of Eq.(3) which is not eventually equal to 1. Then the following statements are true.

- A negative semicycle has at most 4 terms.

- Every positive semicycle of length greater than one is followed by a negative semicycle of length one.

- Every positive semicycle of length one is followed by a negative semicycle of length greater than one.

- Every negative semicycle of length one is followed by a positive semicycle of length one.

- A negative semicycle of length 2,3 or 4 is followed by a positive semicycle with more than one term. 
- If a positive semicycle consists of two terms, they are in decreasing order.

- If a positive semicycle consists of three terms they are in increasing order.

- If the positive semicycle consists of more than three terms, the first term is smaller than the second one and after the first term the terms are decreasing. Either the solution stays above 1 forever, and it is decreasing, or it is just decreasing up to the second to the last term.

- The terms of a negative semicycle of length two are in decreasing order.

।

- The terms of a negative semicycle of length three are in increasing order.

- The first and the last two terms of a negative semicycle of length four are in increasing order and the second and third term are in decreasing order.

The proof is along the lines of the proof of Lemma 3 of Eq.(1) and will be omitted.

\subsection{Global Asymptotic Stability of the Equilibrium of Eq.(3)}

Theorem 3 The positive equilibrium 1 of Eq.(3) is globally asymptotically stable.

Proof Eq.(3) seems to be different from Eqs.(1) and (2) in the sense that here we are unable to find a specific pattern of semicycles which repeats. The local stability of $\bar{x}=1$ follows from linearized stability but for the global attractivity of the equilibrium we will develop a new technique. 
From Lemma 8 and Lemma 9 (ii) and (iv) it is easy to show that

$$
\begin{aligned}
& x_{n+1}<x_{n-2} \Leftrightarrow x_{n-2}>1, \\
& \frac{1}{x_{n+1}}<x_{n-2} \Leftrightarrow x_{n-2}>1, \\
& x_{n+1}>x_{n-2} \Leftrightarrow x_{n-2}<1, \\
& \frac{1}{x_{n+1}}>x_{n-2} \Leftrightarrow x_{n-2}<1, \\
& x_{n+1}=x_{n-2} \Leftrightarrow x_{n-2}=1, \\
& \frac{1}{x_{n+1}}=x_{n-2} \Leftrightarrow x_{n-2}=1 .
\end{aligned}
$$

Next we introduce the following notation:

$$
\tilde{x}_{n}=\left\{\begin{array}{l}
x_{n} \text { if } x_{n} \geq 1, \\
\frac{1}{x_{n}} \text { if } x_{n}<1 .
\end{array}, n=0,1, \ldots\right.
$$

Because of the above properties every solution $\left\{x_{n}\right\}_{n=0}^{\infty}$ of Eq.(3) consists of three subsequences, such that for each $j=0,1,2$ and for all $s \geq 0$

either

$$
\tilde{x}_{3(s+1)+j}<\tilde{x}_{3 s+j}
$$

or

$$
\tilde{x}_{3(s+1)+j}=\tilde{x}_{3 s+j}
$$

Hence there exist numbers $U_{0}, U_{1}, U_{2} \in[1, \infty)$ such that

$$
\lim _{s \rightarrow \infty} \tilde{x}_{3 s+j}=U_{j} \geq 1, \text { for } j=0,1,2 .
$$

Now the terms of each of the three subsequences $\left\{x_{3 s+j}\right\}_{s=0}^{\infty}$, for $j=0,1$, or 2 , are eventually less than 1 , or eventually greater than or equal to 1 , or consist of a aubsequence of terms less than 1 and a subsequence of terms greater than or equal to 
1. Clearly, for $j=0,1$, or 2 any subsequence of $\left\{x_{3 s+j}\right\}_{s=0}^{\infty}$ with terms greater than or equal to 1 converges to $U_{j}$ and any subsequence of $\left\{x_{3 s+j}\right\}_{s=0}^{\infty}$ with terms less than 1 converges to $1 / U_{j}$.

With respect to whether a term of a solution belongs to a positive or a negative semicycle, there exist $2^{6}=64$ distinct patterns that any six consecutive terms of a solution can fall into. For example one pattern is for all six consecutive terms to lie in a positive semicycle. Another pattern is for the first five of the six terms to lie in a. positive semicycle and the sixth term to lie in the following negative semicycle, etc. Clearly one of the 64 patterns has to repeat infinitely often. That is there must exist a subsequence of the solution of the form:

$$
\ldots, x_{n_{s-1}+5}, \underbrace{x_{n_{s}}, x_{n_{s}+1}, x_{n_{s}+2}, x_{n_{s}+3}, x_{n_{s}+4}, x_{n_{s}+5}}_{\text {six consecutive terms }}, x_{n_{s+1}}, \ldots
$$

with the property that for each $j=0,1, \ldots, 5$ all terms of the subsequence

$$
\left\{x_{n_{s}+j}\right\}_{s=0}^{\infty}
$$

lie in positive semicycles or all terms lie in negative semicycles. Furthermore, without loss of generality, we may (and do ) assume that the parity of all these terms is the same.

Now, for each $j=2,3,4$ taking limits, as $s \rightarrow \infty$, in each of the following three equations

$$
x_{n_{s}+1+j}=f\left(x_{n_{s}+j}, x_{n_{s}+j-1}, x_{n_{s}+j-2}\right), s=0,1, \ldots,
$$

and by using the strong negative feedback property of $f$ (in the case of equality), we conclude that $U_{1}, U_{2}$, and $U_{3}$ are all equal to 1 . From this it follows that 1 is a global attractor and the proof is complete. 


\section{A General Result}

The main result in this section is the following theorem.

Theorem 4 Assume that the function $f$ in Eq.(4) satisfies the strong negative feedback property with respect to the variable $x_{i_{0}}$. Then the positive equilibrium $\bar{x}$ of Eq.(4) is globally asymptotically stable.

Proof It is easy to show that the following are true.

- If $x_{i_{0}}>\bar{x} \Rightarrow f\left(x_{0}, x_{1}, \ldots, x_{k}\right) \in\left(\frac{\bar{x}^{2}}{x_{i_{0}}}, x_{i_{0}}\right)$;

- if $x_{i_{0}}=\bar{x} \Rightarrow f\left(x_{0}, x_{1}, \ldots, x_{k}\right)=x_{i_{0}}=\bar{x}$

- if $x_{i_{0}}<\bar{x} \Rightarrow f\left(x_{0}, x_{1}, \ldots, x_{k}\right) \in\left(x_{i_{0}}, \frac{\bar{x}^{2}}{x_{i_{0}}}\right)$.

From this it follows directly that $\bar{x}$ is stable.

Next we introduce the following notation:

$$
\tilde{x}_{n}=\left\{\begin{array}{l}
x_{n} \text { if } x_{n} \geq \bar{x}, \\
\frac{\bar{x}^{2}}{x_{n}} \text { if } x_{n}<\bar{x} .
\end{array}, n=0,1, \ldots\right.
$$

Because of the above properties, for every solution $\left\{x_{n}\right\}_{n=0}^{\infty}$ of Eq.(4) and for each $s \geq 0$, and $j=0,1, \ldots, i_{0}$,

either

$$
\tilde{x}_{\left(i_{0}+1\right)(s+1)+j}<\tilde{x}_{\left(i_{0}+1\right) s+j}
$$

or

$$
\tilde{x}_{\left(i_{0}+1\right)(s+1)+j}=\tilde{x}_{\left(i_{0}+1\right) s+j} .
$$


Hence there exist numbers $U_{0}, U_{1}, \ldots, U_{i_{0}} \in[\bar{x}, \infty)$ such that

$$
\lim _{s \rightarrow \infty} \tilde{x}_{\left(i_{0}+1\right) s+j}=U_{j} \geq \bar{x}, \text { for } j=0,1, \ldots, i_{0} .
$$

Now the terms of each of these subsequences $\left\{x_{\left(i_{0}+1\right) s+j}\right\}_{s=0}^{\infty}$ for $j=0,1, \ldots, i_{0}$ are eventually less than $\bar{x}$, or eventually greater than or equal to $\bar{x}$, or consist of a subsequence of terms less than $\vec{x}$ and a subsequence of terms greater than or equal to $\bar{x}$. Clearly, for $j=0,1, \ldots, i_{0}$ any subsequence of $\left\{x_{\left(i_{0}+1\right) s+j}\right\}_{s=0}^{\infty}$ with terms greater than or equal to $\bar{x}$ converges to $U_{j}$ and any subsequence of $\left\{x_{\left(i_{0}+1\right) s+j}\right\}_{s=0}^{\infty}$ with terms less than $\bar{x}$ converges to $\bar{x}^{2} / U_{j}$.

There exist $2^{k+i_{0}+2}$ distinct patterns that any $\left(k+i_{0}+2\right)$ consecutive terms of a solution can fall into. Clearly one of these patterns has to repeat infinitely often. That is there exists a subsequence of the solution of the form

$$
\ldots, x_{n_{s-1}+k+i_{0}+1}, \underbrace{x_{n_{s}}, x_{n_{s}+1} \ldots, x_{n_{s}+k+i_{0}+1}}_{k+i_{0}+2 \text { consecutive terms }}, x_{n_{s+1}}, \ldots
$$

with the property that for each $j=0,1, \ldots,\left(k+i_{0}+1\right)$ all terms of the subsequence

$$
\left\{x_{n_{s}+j}\right\}_{s=0}^{\infty}
$$

lie in positive semicycles or all terms lie in negative semicycles. As before we may furthermore assume that all the terms are of the same parity.

Now for each $j=k, k+1, \ldots,\left(k+i_{0}\right)$ by taking limits, as $s \rightarrow \infty$, in each of the following $\left(i_{0}+1\right)$ equations

$$
x_{n_{s}+1+j}=f\left(x_{n_{s}+j}, \ldots, x_{n_{s}+j-k}\right), s=0,1, \ldots,
$$


and by using the strong negative feedback property of $f$ (in the case of equality), we conclude that

$$
\bar{x}=U_{1}=U_{2}=\ldots=U_{i_{0}},
$$

and the proof is complete. 


\section{Applications}

In addition to the functions in Eqs.(1), (2) and (3) there are many others satisfying the strong negative feedback property with respect to a variable in the function. We just mention a few of them. The initial conditions are assumed to be positive.

(a) $f(x)=\frac{x^{k}+x^{k-1}+1}{x^{k+1}+x^{k-1}+1}, k \in\{1,2, \ldots\}$,

with respect to the variable $x$.

(b) $f(x, y)=\frac{x+y}{x^{2}+y}$

with respect to the variable $x$.

(c) $f(x, y)=\frac{x+y}{x y+1}$

with respect to the variable $x$.

(d) $f(x, y, z)=\frac{x+y+z}{x^{2}+y+z}$

with respect to the variable $x$.

(e) $f(x, y, z)=\frac{x+y+z}{x+y^{2}+z}$

with respect to the variable $y$.

(f) $f(x, y, z)=\frac{x+y+z}{x+y+z^{2}}$,

with respect to the variable $z$.

(g) $f(x, y, z)=\frac{x+y+z}{x y+z+1}$,

with respect to the variable $x$.

These examples can be extended to higher dimensions in a similar way. 


\section{References}

[1] G.Ladas, Open Problems and Conjectures, Journal of Difference Equations and Applications 4.1 (1998). 
MANUSCRIPT III 


\title{
BOUNDEDNESS OF SOLUTIONS OF A PLANT-HERBIVORE
}

\section{SYSTEM}

\author{
Abstract \\ We investigate the boundedness character of solutions of a Plant-Herbivore \\ System.
}




\section{INTRODUCTION}

In this note we investigate the boundedness character of solutions of the following Plant-Herbivore System

$$
\left.\begin{array}{l}
x_{n+1}=\frac{\alpha x_{n}}{\beta x_{n}+e^{y_{n}}} \\
y_{n+1}=\gamma\left(x_{n}+1\right) y_{n}
\end{array}\right\} \quad, n=0,1, \ldots
$$

where $\alpha \in(1, \infty), \beta \in(0, \infty)$, and $\gamma \in(0,1)$ and the initial conditions $x_{0}$ and $y_{0}$ are arbitrary positive real numbers. This system was introduced and studied by Allen, Hannigan, and Strauss [1]. The model describes the interaction of the grape vine and the adult apple twig borer. A thorough description of the model can be found in [2].

System (1.1) has the equilibrium points $E_{1}=(0,0), E_{2}=\left(\frac{\alpha-1}{\beta}, 0\right)$ and when

$$
\alpha+\beta-\frac{\beta}{\gamma}>1
$$

it also has the equilibrium point $E_{3}=\left(\frac{1}{\gamma}-1, \ln \left(\alpha+\beta-\frac{\beta}{\gamma}\right)\right)$. See [3].

If $\alpha+\beta-\frac{\beta}{\gamma} \leq 1$, it is easy to see that every positive solution of System (1.1) is bounded. In fact, in this case it is shown in [1] that the equilibrium $E_{2}$ is globally asymptotically stable. Our goal here is to investigate the boundedness of solutions when (1.2) holds. 


\section{MAIN RESULT}

In this section we show that when (1.2) holds every positive solution of System (1.1) is bounded. The first result shows that $\left\{x_{n}\right\}$ is bounded.

Theorem 2.1 Assume that (1.2) holds. Let $\left\{x_{n}\right\},\left\{y_{n}\right\}$ be a positive solution of System(1.1). Then there exists an $N \geq 0$ such that

$$
\text { . } \quad x_{n} \leq \frac{\alpha-1}{\beta} \text { for } n \geq N \text {. }
$$

Proof Set

$$
\mathbf{S}=\left\{(x, y): 0<x \leq \frac{\alpha-1}{\beta} \text { and } y>0\right\}
$$

Now observe that the strip $\mathbf{S}$ is invariant under the map

$$
T(x, y)=\left(\frac{\alpha x}{\beta x+e^{y}}, \gamma(x+1) y\right) .
$$

Indeed if $(x, y) \in \mathbf{S}$, then

$$
\frac{\alpha x}{\beta x+e^{y}}<\frac{\alpha x}{\beta x+1} \leq \frac{\alpha\left(\frac{\alpha-1}{\beta}\right)}{\beta\left(\frac{\alpha-1}{\beta}\right)+1}=\frac{\alpha-1}{\beta}
$$

and so $T(x, y) \in \mathbf{S}$.

Hence every solution of System (1.1) which starts in $\mathbf{S}$ stays in $\mathbf{S}$ and so (2.1) holds. It remains to show that every positive solution of System (1.1) enters S. To this end, assume for the sake of contradiction that 


$$
\left(x_{n}, y_{n}\right) \notin \mathbf{S} \text { for } n \geq 0 .
$$

Then clearly

$$
\frac{1}{\gamma}-1<\frac{\alpha-1}{\beta}<x_{n} \leq \frac{\alpha}{\beta} \quad \text { for } \quad n \geq 0
$$

from which it follows that $\left\{x_{n}\right\}$ is bounded and

$$
y_{n+1}=\gamma\left(x_{n}+1\right) y_{n}>\gamma\left(\frac{1}{\gamma}-1+1\right) y_{n}=y_{n}
$$

That is, $\left\{y_{n}\right\}$ is strictly increasing. Since in this region $\left\{x_{n}\right\}$ is decreasing and as System (1.1) has no equilibrium point outside $\mathbf{S}$, it follows that

$$
\lim _{n \rightarrow \infty} y_{n}=\infty
$$

Hence from the first equation of System (1.1) and the fact that $\left\{x_{n}\right\}$ is bounded we find

$$
\lim _{n \rightarrow \infty} x_{n}=0
$$

which contradicts $(2.3)$.

In the next result we assume that a positive solution $\left\{x_{n}\right\},\left\{y_{n}\right\}$ of System (1.1) lies in $\mathbf{S}$ and show that $\left\{y_{n}\right\}$ is bounded.

Theorem 2.2 Assume that (1.2) holds and that $\left\{x_{n}\right\},\left\{y_{n}\right\}$ is a positive solution of System (1.1) such that 


$$
\left(x_{n}, y_{n}\right) \in \mathbf{S} \quad \text { for } n \geq 0 \text {. }
$$

Then $\left\{y_{n}\right\}$ is bounded.

Proof First we subdivide $\mathbf{S}$ into the following subregions and examine how the solution cycles throughout them.

$$
\begin{aligned}
& A=\left\{(x, y): x \leq \frac{1}{\gamma}-1, y \geq \ln (\alpha-\beta x)\right\}, \\
& B=\left\{(x, y): x \leq \frac{1}{\gamma}-1, y<\ln (\alpha-\beta x)\right\}, \\
& C=\left\{(x, y): x>\frac{1}{\gamma}-1, y \leq \ln (\alpha-\beta x)\right\}, \\
& D_{1}=\left\{(x, y): \frac{1}{\gamma}-1 \leq x \leq \frac{\alpha-1}{\beta}, \ln (\alpha-\beta x)<y \leq \ln \left(\frac{\alpha \gamma+\beta \gamma-\beta}{1-\gamma} x\right)\right\}, \\
& D_{2}=\left\{(x, y): \frac{1}{\gamma}-1 \leq x \leq \frac{\alpha-1}{\beta}, \ln \left(\frac{\alpha \gamma+\beta \gamma-\beta}{1-\gamma} x\right)<y\right\} .
\end{aligned}
$$

If for some $\mathrm{n},\left(x_{n}, y_{n}\right) \in A \cup B$, then $x_{n} \leq \frac{1}{\gamma}-1$ and

$$
y_{n+1}=\gamma\left(1+x_{n}\right) y_{n} \leq y_{n}
$$

Similarly if for some $\mathrm{n},\left(x_{n}, y_{n}\right) \in C \cup D_{1} \cup D_{2}$, then

$$
y_{n+1} \geq y_{n}
$$

Next, if for some $\mathrm{n},\left(x_{n}, y_{n}\right) \in B \cup C$, then

$$
y_{n} \leq \ln \left(\alpha-\beta x_{n}\right)
$$


and so

$$
x_{n+1}=\frac{\alpha x_{n}}{\beta x_{n}+e^{y_{n}}} \geq \frac{\alpha x_{n}}{\beta x_{n}+\alpha-\beta x_{n}}=x_{n} .
$$

Similarly if for some $\mathrm{n},\left(x_{n}, y_{n}\right) \in A \cup D_{1} \cup D_{2}$, then $x_{n+1} \leq x_{n}$.

Finally it is very important to observe that if for some $\mathrm{n},\left(x_{n}, y_{n}\right) \in D_{2}$, then

$$
x_{n+1}<\frac{1}{\gamma}-1
$$

and so the solution will go from $D_{2}$ to $A$ in one iteration.

Let $\mathbf{m}$ be the maximum value of the $y$ component of the images $T(x, y)$ where $(x, y) \in B \cup C \cup D_{1}$, and $T(x, y) \in A \cup D_{2}$. Also let $\mathbf{M}$ be the maximum value of the $y$ component of the images $T(x, y)$ where $y \leq \mathbf{m}$ and $(x, y) \in D_{2}$. It is easy to see that the set

$$
\mathbf{R}=\left\{(x, y): 0 \leq x \leq \frac{1}{\gamma}-1, y \leq \mathbf{M}\right\} \cup\left\{(x, y): \frac{1}{\gamma}-1 \leq x \leq \frac{\alpha-1}{\beta}, y \leq \mathbf{m}\right\}
$$

is invariant under the map $T$ defined by $(2.2)$.

As there are no equilibrium points outside of $\mathbf{R}$, it follows that eventually every solution of System (1.1) enters $\mathbf{I}$ and remains in it. 


\section{REFERENCES}

[1] Allen, L.S., Hannigan, M.K., and Strauss, M.J.. Matlicmatical Analysis of a Model for a Plant-Herbivore System, Bulletin of . Mathematical Biology $55(-1)$ (1993), 847-864.

[2] Allen, L.S., Strauss, M.J., Thorvilson, H.G., and Lipe. W.N., A Preliminary Mathematical Model of the Apple Twig Borer (('oleoptera: Bostrichidae) and Grapes on the Texas High Plains, Ecological Modflling 58 (1.991), 369-382.

[3] Kocic, V.L. and Ladas, G., Global Behvior of Nonlincrar Difference, Equations of Higher Order with Applications. Kluwer Academic Publishers, Dordrecht, 1993. 


\section{Bibliography}

Allen, L.S., Hannigan, M.K., and Strauss, M.J., Mathematical Analysis of a Model for a Plant-Herbivore System, Bulletin of Mathematical Biology 55 (4) (1993), 847864.

Allen, L.S., Strauss, M.J., Thorvilson, H.G., and Lipe, W.N., A Preliminary Mathematical Model of the Apple Twig Borer ( Coleoptera: Bostrichidae) and Grapes on the Texas High Plains, Ecological Modelling 58 (1991), 369-382.

Clark, D. and Lewis, J.T., A Collatz Type Difference Equation. Congressus Numerantium 111 (1995), 129-135.

Kocic, V.L. and Ladas, G., Global Behavior of Nonlinear Differenc Equations of Higher Order with Applications. Kluwer Academic Publishers, Dordrecht, 1993.

Ladas, G., Open Problems and Conjectures, Journal of Difference Equations and Applications 2 (1) (1996), 97-99.

Ladas, G., Open Problems and Conjectures, Journal of Difference Equations and Applications 4.1 (1998).

Lagarias, J.C., The $(3 \mathrm{x}+1)$ Problem and its Generalizations. American Mathematical 
Monthly 92 (1985), 3-23. 